\title{
CODE STEMI Program Improves Clinical Outcome in ST Elevation Myocardial Infarction Patients: A Retrospective Cohort Study
}

This article was published in the following Dove Press journal: Open Access Emergency Medicine

\author{
Eka Ginanjar (D) \\ Amal C Sjaaf ${ }^{2}$ \\ Idrus Alwi' \\ Wahyu Sulistyadi ${ }^{2}$ \\ Ede Suryadarmawan ${ }^{2}$ \\ Adik Wibowo ${ }^{2}$ \\ Lies Dina Liastuti (iD) ${ }^{3}$ \\ 'Division of Cardiology, Department of \\ Internal Medicine, Faculty of Medicine, \\ Universitas Indonesia, Dr. Cipto \\ Mangunkusumo Hospital, Jakarta, \\ Indonesia; ${ }^{2}$ Department of Health Policy \\ and Administration, Faculty of Public \\ Health, Universitas Indonesia, Depok, \\ West Java, Indonesia; ${ }^{3}$ Department of \\ Cardiology and Vascular Medicine, \\ Faculty of Medicine, Universitas \\ Indonesia, Jakarta, Indonesia
}

Purpose: One of the most frequent causes of cardiac mortality is ST elevation myocardial infarction (STEMI). Delay in the management of STEMI patients is a cause of high mortality and morbidity. This study aims to determine the effect of the implementation of the CODE STEMI program on major adverse cardiac events (MACE) and mortality of STEMI patients at Dr. Cipto Mangunkusumo General Hospital.

Patients and Methods: This was a retrospective cohort study that enrolled 207 STEMI patients who underwent primary percutaneous coronary intervention (PPCI) in 2015-2018. The patients were divided into two groups. The first group was treated prior to establishing the CODE STEMI program. The other group was treated according to the program, which was implemented in January 2017. Data were collected from medical records, and we retrospectively analyzed all in-hours, MACE, and mortality of STEMI patients from both groups as primary outcomes. Data analysis was done using the Mann-Whitney and chisquare test.

Results: There were 72 and 135 patients in the pre-CODE STEMI and CODE STEMI groups, respectively. D2BT was significantly reduced by $130 \min (288 \pm 306$ vs $158 \pm 81, \mathrm{P}<0.001)$ since the implementation of CODE STEMI program. There were trends to lower in-hospital mortality rates $(8.3 \%$ vs $4.4 \%, \mathrm{RR}=0.53)$ and MACE at 30 days $(48.61 \%$ vs $37.78 \%, \mathrm{RR}=0.77)$.

Conclusion: Implementation of the CODE STEMI program can reduce door-to-balloon time and decrease the MACE and mortality rate in STEMI patients in general hospitals.

Keywords: myocardial infarction, CODE STEMI, percutaneous coronary intervention, major adverse cardiac event, mortality

\section{Introduction}

Cardiovascular diseases (CVDs) are the leading cause of death in the world. ${ }^{1}$ The WHO (World Health Organization) report in 2017 estimated around 17.8 million deaths are caused by cardiovascular disease each year. ${ }^{2}$ Coronary heart disease (CHD) contributed to half of the cases of death from cardiovascular diseases. CHD has various clinical manifestations ranging from asymptomatic to acute conditions, namely acute coronary syndrome (ACS). Acute coronary syndromes include ST elevation myocardial infarction (STEMI), non-ST elevation myocardial infarction (NSTEMI), and unstable angina pectoris (UAP). Based on the Euro Heart Survey in 2004, mortality rates related to STEMI in Europe reached 6-14\%. ${ }^{3}$

The majority of cases in ST elevation myocardial infarction (STEMI) occur mainly because of the occlusion of the main coronary arteries. The extent of
Correspondence: Eka Ginanjar Division of Cardiology, Department of Internal Medicine, Faculty of Medicine Universitas Indonesia - Dr. Cipto

Mangunkusumo General Hospital, Jl.

Diponegoro 7I, Jakarta 10430, Indonesia

Tel +62 8II868093

Email ekginanjar@gmail.com
Open Access Emergency Medicine 2020:12 315-321 
infarction and tissue necrosis in the myocardial depends on the affected blood vessels, compensation by collateral vessels, and the degree and duration of occlusion. ${ }^{4}$ Management of STEMI must be done as soon as possible to prevent further damage to the heart muscles and lower the risk of complications and mortality in patients with STEMI. Reperfusion/revascularization therapy plays a pivotal role in the management of ACS to treat the etiology and improve the clinical condition of patients. ${ }^{5}$ Timely reperfusion with percutaneous coronary intervention (PCI) within 12 hours of symptom onset is currently the treatment of choice in ST-segment-elevation. ${ }^{6}$ Therefore, delay in performing PCI for STEMI patients is the cause of high mortality and the incidence of MACE (major adverse cardiac event).

In general hospitals, the problem that can be found frequently is the lack of health workers' awareness of the importance of immediate treatment for STEMI patients. This problem starts from the Emergency Room (ED). ED crowding has become an issue of great concern in general hospitals, especially since the amount of health workers and facilities are not proportional to the amount of patients that come with different complaints. Moreover, many patients do not want to cooperate easily, adding to the strenuous job of healthcare workers, which resulted in chaotic order in the patients' management, including STEMI patients. There were five potential problems in managing STEMI in daily practices, such as patient delay, diagnosis and treatment decision delay, transportation delay, and lack of collaboration between hospital management and doctors. ${ }^{7}$ All of these resulted in the delay of door-to-balloon time (D2BT), which in turn increased the risk of MACE and mortality.

An establishment of CODE STEMI, an ED physicianactivated STEMI notification system, is expected to help solve this issue. CODE STEMI is a program where a standard integrated clinical pathway is made especially for STEMI patients, including a policy that monitors every incident in handling STEMI patients. The aim is to shorten the $\mathrm{D} 2 \mathrm{BT}$, the time needed for patients to receive immediate reperfusion therapy (primary percutaneous coronary intervention) from initial arrival at the hospital. Previous studies have proven that reducing door-to-balloon time in STEMI is an important strategy for prognosis and is a key performance indicator. ${ }^{8}$

On January 1st, 2017, the Integrated Heart Center team and emergency team of Cipto Mangunkusumo National General Hospital established a standardized CODE STEMI program. The aim of this study was to identify the effect of applying the CODE STEMI protocol to in-hospital clinical outcomes such as MACE and mortality in STEMI patients.

\section{Materials and Methods Study Design and Population}

This was a quantitative, interventional, retrospective cohort study. This study was conducted at Cipto Mangunkusumo National General Hospital in Jakarta. The medical records of all patients with a diagnosis of STEMI and who underwent PPCI from January 1st, 2015 to December 31st, 2018 were reviewed. Patients with MACE and severe comorbidity at initial presentation (acute stroke, hepatic cirrhosis, chronic inflammation disease sepsis, autoimmune, malignancy) were excluded from the study. Patients with incomplete medical record information were also excluded. Using the sample size formula for cohort study, we found the minimum amount of the sample to be 128 subjects. The total number of eligible patients in this study was 207, which was further classified into two groups. The first group was the pre-CODE STEMI group, which consisted of patients presented from 2015 to 2016, and the second group was the CODE STEMI group, consisting of eligible patients from 2017 to 2018. This retrospective study was approved by the Health Research Ethics Committee - University of Indonesia and Cipto Mangunkusumo Hospital. Patient consent to review their medical records was waived by the ethics committee, as consent would be impractical and difficult to obtain. This study was conducted in accordance with the Declaration of Helsinki, and all patient data were maintained with confidentiality.

\section{Data Collection and Processing}

Data were obtained from the hospital's medical record database. We reviewed records from patients' admissions in emergency departments until the end of the hospitalization period. In this program, all patients who came to the ED will go through triage first. Patients will then be examined by an ED physician, and if typical chest pain and ST elevation on an electrocardiogram reading is found, CODE STEMI will be activated. The ED physician will call the on-call cardiologist to confirm the STEMI diagnosis, then alert the interventional cardiologist, catheterization laboratory team, and administrative staff simultaneously. The patient will then be transferred immediately to the catheterization laboratory to get PCI. All steps are regulated meticulously, and each team has a clearly 
defined role in the protocol. The application of this program is supervised closely and evaluated every three months. The CODE STEMI protocol is shown in Figure 1.

The primary end points were MACE (including stroke, cardiogenic shock, congestive heart failure, lethal dysrhythmia, and pericarditis) and mortality. We also measured median D2BT from the patient's arrival at the emergency department to the first balloon inflation. In 2017, the European Heart Society guideline changed the term "door-to-balloon" time to "diagnosis-to-wirecrossing" time as a clinical performance measure. ${ }^{5}$ However, in this study, we still adhere to the old terminology to maintain data consistency from the pre-CODE STEMI era. We compared the MACE rate, mortality rate, and median D2BT time for the patients presenting in the

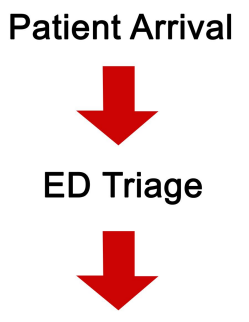

Assessment by ED Physician

Typical chest pain, ST elevantion on ECG

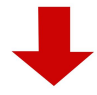

Contact Cardiologist Consultant Confirm STEMI diagnosis to activate CODE STEMI

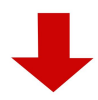

ALERT

1. Interventional cardiologist

2. Laboratory team

3. Clerical and administration staff

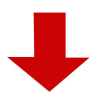

Patient Transfer to Catheterization Laboratory

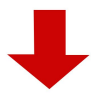

Primary $\mathrm{PCl}$

Figure I CODE STEMI protocol in Cipto Mangunkusumo Hospital. Step-by-step process of STEMI management. Once the CODE STEMI is activated, physician alert the interventional cardiologist, cardiac catheterization laboratory team, and administrative staff simultaneously to hasten the preparation of primary percutaneous intervention.

Abbreviations: ECG, electrocardiogram; ED, emergency department; $\mathrm{PCl}$, primary cutaneous intervention; STEMI, ST elevation myocardial infarction.
pre-CODE STEMI period with the patients presenting in the CODE STEMI period. We also evaluated secondary outcomes including length of stay in hospital and total hospital cost. Total hospital cost is converted from Indonesian Rupiah (IDR) to US Dollar (USD) currency.

\section{Data Analysis}

The data were coded and entered into an Excel datasheet and analyzed using SPSS version 20.0 (IBM, Armonk, NY, United States of America). A univariate analysis was conducted initially and for selected variables, and bivariate analysis was conducted subsequently. We used the chisquare $\left(\mathrm{x}^{2}\right)$ test to compare the MACE and mortality rates between each group. To identify the correlation between CODE STEMI with door-to-balloon time and the secondary outcomes, we used the Mann-Whitney test. A 95\% confidence interval was used for all normally distributed data, while median and interquartile ranges were used for nonnormally distributed data. We also calculated the relative odds ratio (ROR) for MACE and Mortality outcome as a quantitative measure for selection bias.

\section{Results}

\section{Patient Characteristics}

We found a total of 358 medical records of patients presented with STEMI who underwent PPCI between January 2015 and December 2018. Of those, 50 medical records are incomplete and 101 patients with complete medical records had severe comorbidities at initial presentation, thus were excluded. There were 207 patients who met the inclusion criteria and were studied retrospectively. The cohort was divided into two groups. The first group consisted of 72 patients who were treated before the establishment of the CODE STEMI program (mean age of 59.7; 87\% were men). The second group consisted of 135 patients treated according to the CODE STEMI program that was initiated in January 2017 (mean age of 56.1; 86\% were men). Risk factors for cardiovascular disease such as diabetes mellitus, hypertension, dyslipidemia, obesity, acute kidney failure, and chronic kidney failure were distributed evenly in both the CODE STEMI group and the pre-CODE STEMI group. The baseline and clinical characteristics in both groups were similar, with no discernible discrepancies (Table 1).

\section{Door-to-Balloon Time}

In the pre-CODE STEMI group, the median D2BT was 288 min (interquartile range [IQR] 120-1376). On the other hand, 
Table I Baseline Characteristics of the Patients

\begin{tabular}{|c|c|c|c|}
\hline Characteristics & $\begin{array}{l}\text { CODE } \\
\text { STEMI } \\
(\mathrm{N}=135)\end{array}$ & $\begin{array}{l}\text { Pre CODE } \\
\text { STEMI }(N=72)\end{array}$ & $P$ value \\
\hline $\begin{array}{l}\text { Sex, } n(\%) \\
\text { Male } \\
\text { Female }\end{array}$ & $\begin{array}{l}116(85.9) \\
19(14.1)\end{array}$ & $\begin{array}{l}63(87.5) \\
9(12.5)\end{array}$ & 0.75 \\
\hline $\begin{array}{l}\text { Age } \\
\text { Risk Factor }\end{array}$ & $\begin{array}{l}56.1 \\
55(40.7)\end{array}$ & $\begin{array}{l}59.7 \\
34(47.2)\end{array}$ & $\begin{array}{l}0.031 \\
0.3696\end{array}$ \\
\hline $\begin{array}{l}\text { Diabetes Mellitus, n (\%) } \\
\text { Hypertension, n (\%) } \\
\text { Dyslipidemia, n (\%) } \\
\text { Obesity, n (\%) } \\
\text { Acute Kidney Injury, n (\%) } \\
\text { Chronic Kidney Disease, } \\
\text { n (\%) }\end{array}$ & $\begin{array}{l}74(54.81) \\
51(37.8) \\
9(6.7) \\
23(17.0) \\
18(13.43)\end{array}$ & $\begin{array}{l}41(56.94) \\
24(33.3) \\
2(28) \\
16(22.5) \\
22(30.56)\end{array}$ & $\begin{array}{l}0.7690 \\
0.526 \\
0.2348 \\
0.3384 \\
0.0031\end{array}$ \\
\hline $\begin{array}{l}\text { GRACE Score, } \mathrm{n}(\%) \\
\text { Severe } \\
\text { Moderate } \\
\text { Mild }\end{array}$ & $\begin{array}{l}4(21.05) \\
3(15.79) \\
12(63.16)\end{array}$ & $\begin{array}{l}3(18.92) \\
3(16.22) \\
12(66.67)\end{array}$ & 0.944 \\
\hline $\begin{array}{l}\text { Onset, } \mathrm{n}(\%) \\
>6 \text { hours } \\
\leq 6 \text { hours }\end{array}$ & $\begin{array}{l}22(16) \\
23(17)\end{array}$ & $\begin{array}{l}55(76.3) \\
68(94)\end{array}$ & 0.631 \\
\hline
\end{tabular}

the CODE STEMI group had a significantly lower D2BT, with a median of $158 \mathrm{~min}$ (IQR 66-640), representing a 130 $\min (45 \%)$ improvement $(\mathrm{p}<0.01)$. Figure 2 shows a decreasing trend of D2BT each year after the implementation of CODE STEMI.

\section{In-Hospital Clinical Outcomes}

Data analysis results showed no significant difference between the two groups in MACE and mortality rate.

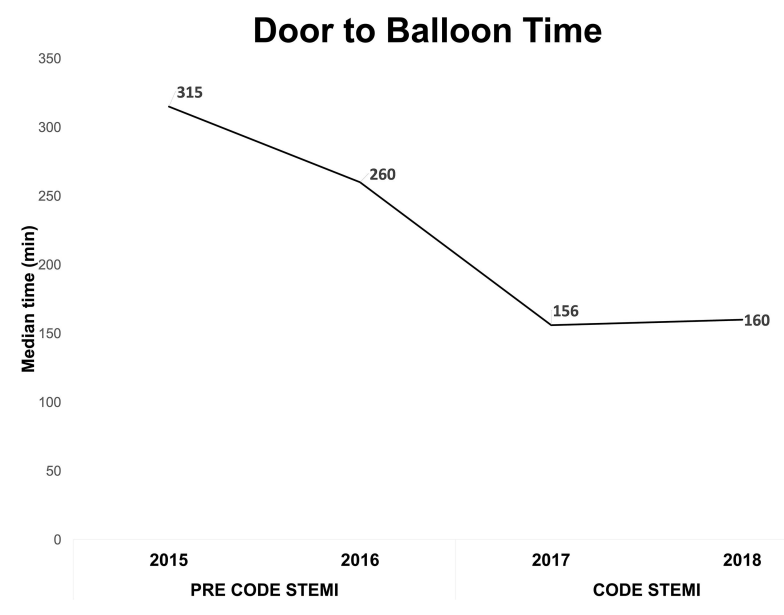

Figure 2 Door to balloon time trend in STEMI patients by year.
Table 2 Data Analysis: MACE and Mortality

\begin{tabular}{|l|l|l|l|}
\hline & $\begin{array}{l}\text { CODE } \\
\text { STEMI } \\
\mathbf{n}=135\end{array}$ & $\begin{array}{l}\text { Pre CODE } \\
\text { STEMI } \\
\mathbf{n}=\mathbf{7 2}\end{array}$ & RR (95\% CI) \\
\hline MACE & $5 I(37.78 \%)$ & $35(48.6 I \%)$ & $0.777(0.564-I .07 I)$ \\
Mortality & $6(4.44 \%)$ & $6(8.33 \%)$ & $0.533(0.178-I .593)$ \\
\hline
\end{tabular}

Abbreviations: $\mathrm{Cl}$, confidence Interval; D2BT, door to balloon time; MACE, major adverse cardiovascular events; RR, risk ratio.

However, as seen in Table 2, we noticed a reduction of inhospital MACE and mortality rate in the CODE STEMI group. There is a decrease in MACE incidence in the period after CODE STEMI was implemented, with a risk ratio of 0.77 . The MACE rate was reduced by $10.83 \%$ from $48.61 \%$ in the pre-CODE STEMI period to $38.78 \%$ after the implementation of the CODE STEMI program. The most common MACE that can be found in both groups is heart failure. We can also see a reduction in mortality from $8.33 \%$ in the pre-CODE STEMI group to $4.44 \%$ in the CODE STEMI group. The decrease in the percentage of these two parameters was not statistically significant, but clinically showed a decrease in the incidence of MACE and mortality in the group of patients who were treated using the STEMI CODE program $(\mathrm{RR}=0.777$ and 0.533 , respectively). Relative odds ratio (ROR) computed as the ratio of the odds ratio among participants to the corresponding estimate in the source population. The ROR is 0.79 and 0.5 for MACE and mortality, respectively.

\section{Secondary Outcomes}

The difference in length of stay for 1 day was statistically significant $(\mathrm{p}=0.009)$. The median length of stay in the CODE STEMI group was 6 days with a standard deviation of 3.8 days, whereas for the Pre CODE STEMI group 7 days with a standard deviation of 3.5 days. Starting from 2017, the median length of stay of STEMI patients are maintained in 6 days, while the 2015 and 2016 median length of stay is 7.5 and 6.5 days, respectively. For total hospital cost, a significant difference of USD 1027.16 (21\%) was found between the total costs of the two groups (Table 3). The average hospital bill in the CODE STEMI group was USD 3,818.95, while for the Pre CODE STEMI group was USD 4870.97. There is a declining trend of total hospital cost each year from 2015 to 2018 especially after the implementation of CODE STEMI, from 5066 USD in 2015, 4746 USD in 2016, 4278 USD in 2017, to 3589 USD in 2018. 
Table 3 Data Analysis: Door to Balloon Time, Total Hospital Cost, Length of Stay

\begin{tabular}{|c|c|c|c|}
\hline Variable & $\begin{array}{l}\text { CODE STEMI } \\
n=135\end{array}$ & $\begin{array}{l}\text { Pre CODE STEMI } \\
\mathrm{n}=72\end{array}$ & $\mathbf{P}$ \\
\hline $\begin{array}{l}\text { D2BT, minutes } \\
\text { (minimum-maximum) }\end{array}$ & $158(66-640)$ & $288(120-1376)$ & $<0.001$ \\
\hline $\begin{array}{l}\text { Total hospital cost, USD } \\
\text { (minimum-maximum) }\end{array}$ & $\begin{array}{l}3818.95 \\
(543.03-12,172.11)\end{array}$ & $\begin{array}{l}4870.97 \\
(1690.55-16,828.63)\end{array}$ & $<0.001$ \\
\hline $\begin{array}{l}\text { Length of stay, days } \\
\text { (minimum-maximum) }\end{array}$ & $6(2-34)$ & $7(2-24)$ & 0.009 \\
\hline
\end{tabular}

Abbreviations: D2BT, door to balloon time; USD, US dollar.

\section{Discussion}

We found that the introduction of CODE STEMI at our institution has successfully improved D2BT and reduced inhospital MACE and mortality. In our study, the implementation of CODE STEMI resulted in a $45 \%(130 \mathrm{~min})$ improvement of D2BT, from $288 \mathrm{~min}$ to $158 \mathrm{~min}$. This result is similar to a study in Australia and Saudi Arabia, which showed a $22.1 \mathrm{~min}$ and 30.5 min reduction of D2BT in the post-CODE STEMI period. ${ }^{8,9}$ Previous studies have revealed that prolonged D2BT for patients with STEMI was associated with increased mortality. ${ }^{10-12}$ The target of D2BT in STEMI patients undergoing PPCI according to American Heart Association and European Society of Cardiology (ESC) is $\leq 90 \mathrm{~min}^{13}$ Although we have not reached the international standards, the CODE STEMI program has significantly reduced the door-to-balloon time, since even a small reduction in D2BT has become critically important in reducing mortality risk. ${ }^{14}$ The D2BT of less than 90 minutes has been shown to be associated with a reduction in major in-hospital complications. ${ }^{15,16}$

We were able to observe a decrease in the incidence of MACE in STEMI patients by $10.83 \%$ in the CODE STEMI group with a risk ratio of 0.78 . This means that there is a 0.78 risk of MACE in STEMI patients who are not treated using the CODE STEMI protocol. Although the decrease was not proven to be statistically significant, these results still show a downward trend in the incidence of MACE in STEMI patients, which is still an important value in clinical practice. These results are consistent with one study that reported a non-statistically significant decrease in the incidence of 30-day MACE (5\% reduction) and 12-month MACE (10.1\% reduction) by the activation of the CODE STEMI program. ${ }^{8}$ Another study in 2018 also reported lower complications in patients treated with the CODE STEMI program, specifically the incidence of recurrent infarction, which drops to $8 \%$. $(p=0.043){ }^{9}$
In alignment with MACE, there was a decrease in mortality by around $4 \%$ after the implementation of the CODE STEMI program, with a risk ratio of 0.53 . Clinically, this means there is a reduced risk of mortality in STEMI patients treated using the CODE STEMI program. A study conducted in Canada also showed a decrease in hospital mortality from $10 \%$ to $4.7 \%$ in the first year the CODE STEMI was applied. ${ }^{17}$ Every minute of delay in primary angioplasty for STEMI affects oneyear mortality, even after adjustment for baseline characteristics. $^{18}$ Both results mean that the CODE STEMI program can reduce risk of mortality and MACE in STEMI patients. This is closely related to the reduction of D2BT after CODE STEMI was implemented. Shorter D2BT, which prompts early revascularization, has been associated with improved clinical outcomes in STEMI patients. $^{14,15,16}$

The one day reduction in length of stay of STEMI patients after the implementation of CODE STEMI protocol is consistent with a study in a teaching hospital in New Jersey, which found that during the first year CODE STEMI implementation, the average length of stay of patients dropped from 4 days to 3 days. ${ }^{13}$ Other study from Le May also stated that 1-year implementation of CODE STEMI as a city-wide protocol in Ottawa successfully reduced hospital length of stay to only 4 days.

The reduction of MACE, mortality, and length of stay contributes to the reduction of total hospitalization cost of STEMI patients. Lower treatment cost is required when patients do not have to go through extensive treatment and stay too long in the hospital after PPCI. Our study found that the implementation of CODE STEMI protocol has successfully reduced total hospital cost up to $21 \%$.

Providing timely emergency PCI is a complex undertaking demanding rapid coordination of care by multiple physicians, nurses, and hospital staff. In 2006, the D2B alliance was launched, listing emergency physician-initiated CODE 
STEMI as a method to reduce the door-to-balloon time. ${ }^{1,2}$ The success of the CODE STEMI program in reducing D2BT, thus reducing the in-hospital MACE and mortality in our institution, was made possible by changing the system that previously required a step-by-step serial process to a parallel process system with simultaneous activation of the catheterization laboratory, the cardiology on call, the interventional cardiologist on call, and the administrative and clerical staff. This strategy has been shown to reduce transfer time from the ED to the catheterization laboratory and the arrival of catheterization laboratory team.

Since our study was a cohort retrospective study, we are aware that selection bias may occur during identification of the study population. Therefore, we made sure to select patients using rigorous criteria to avoid confounding results. Patients in both groups are also originated from the same population which is STEMI patients who underwent PCI in 2015-2018. However, we did found that ROR from both mortality and MACE calculation is less than one, which might indicate a possible negative bias (underestimation) for the result.

In our study, the mean age of presentation was 57 years, which is almost a decade earlier than that reported in several other studies. ${ }^{17,18}$ It highlights the fact that people tend to get STEMI at a younger age these days, especially in our country. Moreover, our patients have a high prevalence of risk factors for coronary artery diseases, especially hypertension, diabetes mellitus, and dyslipidemia. This emphasizes the increased incidence of metabolic syndrome as a risk factor of atherosclerosis, which is the main cause of most STEMI. Therefore, a well-designed program to deal with the consequences is very much needed.

Although the number of patients included in this study is relatively small and there are still some potential biases in the study, the trends in favorable outcomes are encouraging and generalizable to most healthcare organizations, especially for general hospitals in developing countries. A larger sample size might be able to reveal a significant difference in the in-hospital MACE and mortality between the two groups.

\section{Limitation}

Our study had several limitations. First of all, it was a retrospective cohort study without randomization to either CODE STEMI or pre-CODE STEMI groups. Our study was also a single-center study with data collected specific to our institution, so the results might not reflect the performance of other general hospitals. Follow up was only done during patient's hospitalization and 30 days post PCI procedure, so the incidence of MACE and mortality after that period was undocumented. Furthermore, there might be confounding factors that could affect the clinical outcomes between 2015 and 2018, such as physician and interventional cardiologist clinical experience and skills which can improve throughout the years.

\section{Conclusion}

The implementation of the CODE STEMI program in general hospitals was associated with a significant reduction in D2BT and reduced MACE and mortality rate in STEMI patients. STEMI patients treated without the use of the CODE STEMI program have increased risk of both MACE and mortality incidence. Furthermore, CODE STEMI may also be considered as an optimal intervention for STEMI patients for its ability to provide an effective treatment with the lowest cost. This re-emphasizes the significance of a systematic program with early catheterization laboratory activation and concomitant early reperfusion in patients with STEMI, especially in general hospitals.

\section{Disclosure}

The authors report no conflicts of interest for this work.

\section{References}

1. WHO. Non Communicable Diseases Country Profiles 2011. Vol. ISBN 978 9. Geneva; 2011.

2. Mensah GA, Roth GA, Fuster V. The global burden of cardiovascular diseases and risk factors. $J$ Am Coll Cardiol. 2019;74(20):2529 LP2532. doi:10.1016/j.jacc.2019.10.009.

3. Mandelzweig L, Battler A, Boyko V, et al. The second euro heart survey on acute coronary syndromes: characteristics, treatment, and outcome of patients with ACS in Europe and the Mediterranean Basin in 2004. Eur Heart J. 2006;27(19):2285-2293.

4. Koh JQ, Tong DC, Sriamareswaran R, et al. In-hospital "CODE STEMI" improves door-to-balloon time in patients undergoing primary percutaneous coronary intervention. 'Emerg Med Australas. 2018;30(2):222-227. doi:10.1111/1742-6723.12855

5. Alyahya AA, Alghammass MA, Aldhahri FS, et al. The impact of introduction of Code-STEMI program on the reduction of door-toballoon time in acute ST-elevation myocardial infarction patients undergoing primary percutaneous coronary intervention: A single-center study in Saudi Arabia. J Saudi Hear Assoc. 2018;30 (3):172-179. doi:10.1016/j.jsha.2017.11.002

6. Rathore SS, Curtis JP, Chen J, et al. Association of door-to-balloon time and mortality in patients admitted to hospital with ST elevation myocardial infarction: national cohort study. BMJ. 2009;338:b1807. doi:10.1136/bmj.b1807

7. McNamara RL, Herrin J, Bradley EH, et al. Hospital improvement in time to reperfusion in patients with acute myocardial infarction, 1999 to 2002. J Am Coll Cardiol. 2006;47(1):45-51. doi:10.1016/j. jacc.2005.04.071

8. McNamara RL, Herrin J, Wang Y, et al. Impact of delay in door-toneedle time on mortality in patients with ST-segment elevation myocardial infarction. Am $J$ Cardiol. 2007;100(8):1227-1232. doi:10.1016/j.amjcard.2007.05.043 
9. O'Gara PT, Frederick GK, Deborah DA, et al. ACCF/AHA guideline for the management of ST-elevation myocardial infarction: executive summary. Circulation. 2013;127(4):529-555. doi:10.1161/CIR.0b0 $13 \mathrm{e} 3182742 \mathrm{c} 84$.

10. McNamara RL, Wang Y, Herrin J, et al. Effect of door-to-balloon time on mortality in patients with ST-segment elevation myocardial infarction. J Am Coll Cardiol. 2006;47(11):2180-2186. doi:10.1016/j. jacc.2005.12.072

11. AlHabib KF, Sulaiman K, Al Suwaidi J, et al. Patient and system-related delays of emergency medical services use in acute ST-elevation myocardial infarction: results from the third gulf registry of acute coronary events (Gulf RACE-3Ps. PLoS One. 2016;11:1. doi:10.1371/journal.pone.0147385

12. Shiomi H, Nakagawa Y, Morimoto T, et al. Association of onset to balloon and door to balloon time with long term clinical outcome in patients with ST elevation acute myocardial infarction having primary percutaneous coronary intervention: observational study. BMJ. 2012;344:e3257. doi:10.1136/bmj.e3257

13. Le May M. Code STEMI: implementation of a city-wide program for rapid assessment and management of myocardial infarction. Can Med Assoc J. 2009;181(8):E136--E137. doi:10.1503/cmaj.091087

14. De Luca G, Suryapranata H, Ottervanger JP, Antman EM. Time delay to treatment and mortality in primary angioplasty for acute myocardial infarction: every minute of delay counts. Circulation. 2004;109 (10):1223-1225. doi:10.1161/01.CIR.0000121424.76486.20
15. Cannon CP, Gibson CM, Lambrew CT, et al. Relationship of symptom-onset-to-balloon time and door-to-balloon time with mortality in patients undergoing angioplasty for acute myocardial infarction. JAMA. 2000;283(22):2941-2947. doi:10.1001/ jama.283.22.2941

16. Berger PB, Ellis SG, Holmes DR Jr, et al. Relationship between delay in performing direct coronary angioplasty and early clinical outcome in patients with acute myocardial infarction: results from the global use of strategies to open occluded arteries in Acute Coronary Syndromes (GUSTO-IIb) trial. Circulation. 1999;100(1):14-20. doi:10.1161/01. CIR.100.1.14

17. Vernon ST, Sean C, Mario D, et al. ST-segment-elevation myocardial infarction (STEMI) patients without standard modifiable cardiovascular risk factors - how common are they, and what are their outcomes? J Am Heart Assoc. 2019;8(21):e013296. doi:10.1161/ JAHA. 119.013296

18. Terkelsen CJ, Lassen JF, Nørgaard BL, et al. Mortality rates in patients with ST-elevation vs. non-ST-elevation acute myocardial infarction: observations from an unselected cohort. Eur Heart J. 2004;26(1):18-26.

\section{Publish your work in this journal}

The Open Access Emergency Medicine is an international, peerreviewed, open access journal publishing original research, reports, editorials, reviews and commentaries on all aspects of emergency medicine. The manuscript management system is completely online and includes a very quick and fair peer-review system, which is all easy to use. Visit http://www.dovepress.com/testimonials.php to read real quotes from published authors. 